\title{
Distance-from-the-wall scaling of turbulent motions in wall-bounded flows
}

R. Baidya, J. Philip, N. Hutchins, J. P. Monty, and I. Marusic

Citation: Phys. Fluids 29, 020712 (2017); doi: 10.1063/1.4974354

View online: http://dx.doi.org/10.1063/1.4974354

View Table of Contents: http://aip.scitation.org/toc/phf/29/2

Published by the American Institute of Physics

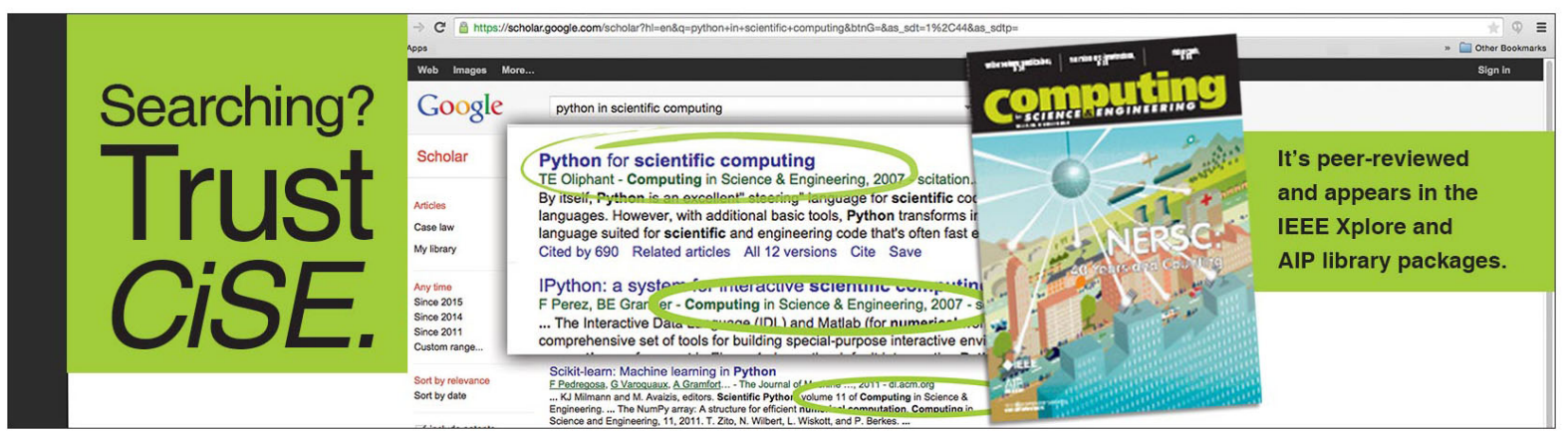




\title{
Distance-from-the-wall scaling of turbulent motions in wall-bounded flows
}

\author{
R. Baidya, J. Philip, N. Hutchins, J. P. Monty, and I. Marusic \\ Department of Mechanical Engineering, University of Melbourne, Melbourne, Victoria 3010, Australia
}

(Received 1 July 2016; accepted 6 October 2016; published online 16 February 2017)

\begin{abstract}
An assessment of self-similarity in the inertial sublayer is presented by considering the wall-normal velocity, in addition to the streamwise velocity component. The novelty of the current work lies in the inclusion of the second velocity component, made possible by carefully conducted subminiature $x$-probe experiments to minimise the errors in measuring the wall-normal velocity. We show that not all turbulent stress quantities approach the self-similar asymptotic state at an equal rate as the Reynolds number is increased, with the Reynolds shear stress approaching faster than the streamwise normal stress. These trends are explained by the contributions from attached eddies. Furthermore, the Reynolds shear stress cospectra, through its scaling with the distance from the wall, are used to assess the wall-normal limits where self-similarity applies within the wall-bounded flow. The results are found to be consistent with the recent prediction from the work of Wei et al. ["Properties of the mean momentum balance in turbulent boundary layer, pipe and channel flows," J. Fluid Mech. 522, 303-327 (2005)], Klewicki ["Reynolds number dependence, scaling, and dynamics of turbulent boundary layers," J. Fluids Eng. 132, 094001 (2010)], and others that the self-similar region starts and ends at $z^{+} \sim \mathcal{O}\left(\sqrt{\delta^{+}}\right)$and $O\left(\delta^{+}\right)$, respectively. Below the self-similar region, empirical evidence suggests that eddies responsible for turbulent stresses begin to exhibit distance-from-the-wall scaling at a fixed $z^{+}$location; however, they are distorted by viscous forces, which remain a leading order contribution in the mean momentum balance in the region $z^{+} \lesssim \mathcal{O}\left(\sqrt{\delta^{+}}\right)$, and thus result in a departure from self-similarity. Published by AIP Publishing. [http://dx.doi.org/10.1063/1.4974354]
\end{abstract}

\section{INTRODUCTION}

A large amount of effort has been invested in understanding wall-bounded turbulent flows, primarily due to its importance in many applications. One of the challenges associated with the study of wall turbulence is that it contains a range of length scales. The smallest scale corresponds to the viscous length scale, $v / U_{\tau}$, where $v$ and $U_{\tau}$ are the kinematic viscosity and friction velocity $\left(U_{\tau}=\left.\sqrt{v /(\partial U / \partial z)}\right|_{z=0}\right)$. Here, $U$ and $z$ are the streamwise mean velocity and wall-normal distance with origin at the wall, respectively, and we shall use $x$ and $y$ for the streamwise and spanwise directions. The largest scale is typically taken to be the turbulent boundary layer thickness $\delta$, or the centreline height for pipe and channel flows. ${ }^{3}$ Another possible choice for a length scale is $z$-the distance from the wall. ${ }^{4,5}$

Turbulent motions are usually expected to scale with one of these three length scales $\left(v / U_{\tau}, z, \delta\right)$ or with the homogeneous turbulence length scales (Kolmogorov length scale and Taylor microscale), or a combination of these. To make the notion of turbulent length scales more precise, it is usual to consider the streamwise spectra $\phi_{i j}\left(k_{x}, z\right)$ of the velocity components at different wall-normal location, where $k_{x}$ is the streamwise wavenumber and $i, j=1,2,3$ or $u, v, w$. In this paper, we shall specifically consider the spectra of $u, w$ velocities and the cospectra of Reynolds shear stress $\left(\phi_{u u}\right.$, $\left.\phi_{w w}, \phi_{u w}\right)$. It should be noted that the integral of $\phi_{u u}, \phi_{w w}$, and $\phi_{u w}$ over $k_{x}$ results in the turbulent stresses $\overline{u^{2}}, \overline{w^{2}}$, and $\overline{u w}$, respectively. Here, capitalisation and overline denote time averaging. In contrast to the multitude of available length scales, for velocity scale, $U_{\tau}$ alone characterises the velocity of the turbulent motions, and + denotes the scaling of velocities with $U_{\tau}$.

The situation is indeed complicated if we want to understand the scaling of $\phi_{i j}\left(k_{x}, z\right)$ with respect to the various length scales over the full range of $k_{x}$ and $z$. However, if we concentrate on arguably the most important wall-normal region in the boundary layer, the inertial sublayer (also known as the logarithmic layer), and the energy containing motions, the situation simplifies, since the effects of viscosity are negligible. Consequently, the viscous length scale $\left(v / U_{\tau}\right)$ does not feature in the dynamics, while the homogeneous length scales (Kolmogorov length scale and Taylor microscale) are found to be relevant only for the fine-scale motion with negligible contribution to the overall turbulent stresses. Hence, the dominant length scales in these cases are $z$ and $\delta$ (some evidence for this will be given in Sec. III). The scaling of turbulent motions with $z$ has been employed in a number of different ways (e.g., attached eddy hypothesis, ${ }^{5,6}$ vortex clusters, ${ }^{7}$ and the $L_{\beta}$ hierarchy ${ }^{8}$ ), and collectively we refer to them as the distance-from-the-wall scaling. We note that the inertial sublayer corresponds to a region that is sufficiently far away both from the wall and the edge of the boundary layer or the centreline in internal flows.

Most attention has been paid to $u$-motions primarily because of its ease in measurement, where Perry, Henbest, and Chong ${ }^{9}$ show that there are two regions in wavenumber space for $\phi_{u u}$, with one scaling with $z$ and the other with $\delta$, and 
TABLE I. Experimental parameters for $\times$-probe measurements.

\begin{tabular}{lcccccccccc}
\hline \hline $\begin{array}{l}x \\
(\mathrm{~m})\end{array}$ & $\begin{array}{c}U_{\infty} \\
\left(\mathrm{ms}^{-1}\right)\end{array}$ & $R e_{\tau}$ & $\begin{array}{c}v / U_{\tau} \\
(\mu \mathrm{m})\end{array}$ & $\begin{array}{c}U_{\tau} \\
\left(\mathrm{ms}^{-1}\right)\end{array}$ & $\begin{array}{c}\delta \\
(\mathrm{m})\end{array}$ & $\Delta t^{+}$ & $T U_{\infty} / \delta$ & $l_{x}^{+}$ & $l_{z}^{+}$ & $\Delta s_{y}^{+}$ \\
\hline 2 & 15.2 & 2600 & 28 & 0.569 & 0.071 & 0.513 & 18000 & 14 & 14 & 7 \\
7 & 15.2 & 5100 & 30 & 0.526 & 0.153 & 0.436 & 19000 & 13 & 13 & 7 \\
18 & 14.8 & 10600 & 32 & 0.484 & 0.338 & 0.377 & 18000 & 12 & 12 & 6 \\
\hline \hline
\end{tabular}

in-between them the overlap region displaying a $k_{x}^{-1}$ spectrum. It is interesting to note that there has been a large effort in searching for the $k_{x}^{-1}$ region, and this has proven to be mostly elusive, mainly because of the need for high Reynolds numbers $(R e)$ coupled with the simultaneous need to make measurements in the inertial sublayer (which physically gets very close to the wall with increasing $R e$ ). In addition, as Davidson, Nickels, and $\mathrm{Krogstad}^{10}$ mention, the $k_{x}^{-1}$ behaviour in the one dimensional spectra is likely contaminated by aliasing from three dimensional effects. Therefore, the $u$-motions show a mixed scaling in $z$ and $\delta$ rather than a pure distance-from-thewall scaling. In contrast, the $w$ motions do not scale with $\delta$, and in this paper we will show that the $w$ and $u w$-motions exhibit collapse in the distance-from-the-wall (or $z$-) scaling more readily compared to the $u$ motions. Furthermore, we shall also provide a phenomenological explanation for the mixed scaling of $\phi_{u u}$ and the pure $z$-scaling of $\phi_{w w}$ and $\phi_{u w}$ using inviscid vortex-eddy calculations with their sizes increasing linearly with distance from the wall. This also enables us to demonstrate how finite and asymptotically high $R e$ affect the scaling of these spectra.

The $u w$-motions are important as they quantify momentum transport across the boundary layer and are central to theories that describe the mean flow. For example, the original theories ${ }^{3,4}$ to derive a logarithmic mean velocity profile $\left(U^{+}=(1 / \kappa) \ln \left(z^{+}\right)+A\right.$, where $\kappa$ is the von Kármán constant and $A$ the geometry dependent intercept) in the inertial sublayer involve assuming that $-\overline{u w}^{+}=l_{\text {mixing }} U_{\tau} d U^{+} / d z^{+}$. Here the mixing length $l_{\text {mixing }}$ is assumed to scale with distancefrom-the-wall, $l_{\text {mixing }}=\kappa z$, which along with the approximation that $-\overline{u w}^{+}=1$ in the inertial sublayer leads to the log law in $U$. Despite the known limitations of the underlying theory, the log law has a strong experimental support and an important consequence of the formulation is that the $u w$-motions must follow $z$-scaling. To date, evidence for this has been unclear. In the rest of the paper, we will give evidence for this $z$-scaling of the $u w$-motions and also show that $\phi_{u w}$ follows the $z$-scaling precisely between the wall-normal locations where $U$ exhibits a $\log$ behaviour. This confirms that $U$ and $-\overline{u w}$ are intimately related to each other via $z$-scaling.

The obvious difficulties with obtaining an unambiguous $z-$ scaling for the $w$ spectra and $u w$ cospectra are the requirement of high $R e$, which in turn leads to difficulties in reaching positions close to the wall, not to mention the issues related with measuring both $u$ and $w$ simultaneously with sufficient accuracy and resolution. In the present case, we overcome these difficulties by carrying out measurements in the High Reynolds Number Boundary Layer Wind Tunnel (HRNBLWT), which has a working section of $27 \mathrm{~m}$ in length, producing a boundary layer of approximately $30 \mathrm{~cm}$ thickness at the measurement station of $x=21 \mathrm{~m}$. The facility is complimented with a new custom-made subminiature $\times$-probe for the simultaneous measurement of $u$ and $w$ to reach close to the wall and still maintain sufficient resolution. ${ }^{11,12}$

\section{BOUNDARY LAYER MEASUREMENT SYSTEMS AND TURBULENT STRESS DISTRIBUTIONS}

All experiments reported in this paper have been conducted in HRNBLWT, ${ }^{13}$ located at the University of Melbourne. Measurements are taken at 2, 7, and $18 \mathrm{~m}$ downstream of the tripped inlet with a nominal free-stream velocity $\left(U_{\infty}\right)$ of $15 \mathrm{~ms}^{-1}$. Here, the friction velocity is only a weak function of the streamwise distance for a fixed free-stream velocity. Therefore, the same probe can be used to measure at different streamwise locations (and hence at different $R e$ ), while still ensuring that the sensor size across multiple $R e$ is matched to within a single viscous unit.

Details of the experimental conditions are given in Table I. The value for $U_{\tau}$ and $\delta$ given in Table I have been obtained by fitting the mean velocity profile to a composite velocity formulation. ${ }^{14}$ The sampling interval $\Delta t$ is chosen so that it is sufficiently low to capture the smallest energetic length scale $^{15}$ (i.e., $\Delta t^{+}<3$ ), while the total sampling time $T$ corresponds to approximately 20000 boundary-layer turnover times $\left(T U_{\infty} / \delta\right)$. Figure 1 shows the custom $\times$-probe used, and its dimensions are $0.4 \times 0.4 \mathrm{~mm}\left(l_{x} \times l_{z}\right)$ in the $x$ and $z$ directions with the wire separation of $0.2 \mathrm{~mm}\left(\Delta s_{y}\right)$ in the $y$ direction.

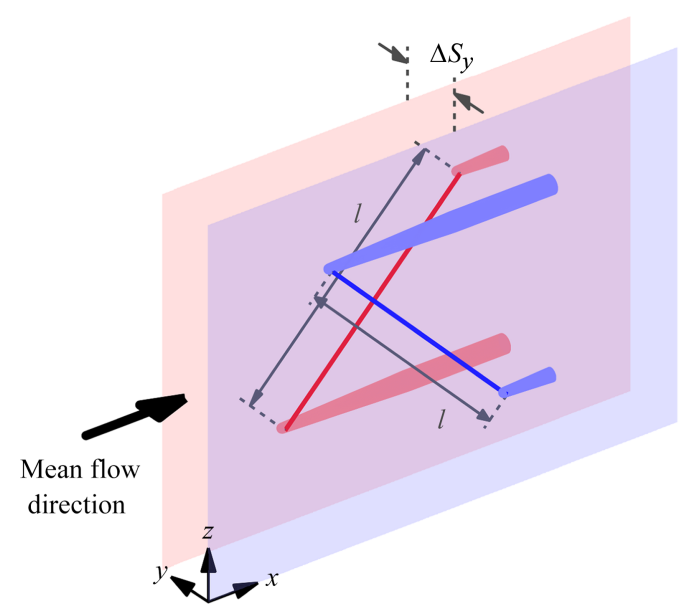

FIG. 1. A schematic of $\times$-probe showing sensor lengths $l$ and spacing $\Delta s_{y}$. 

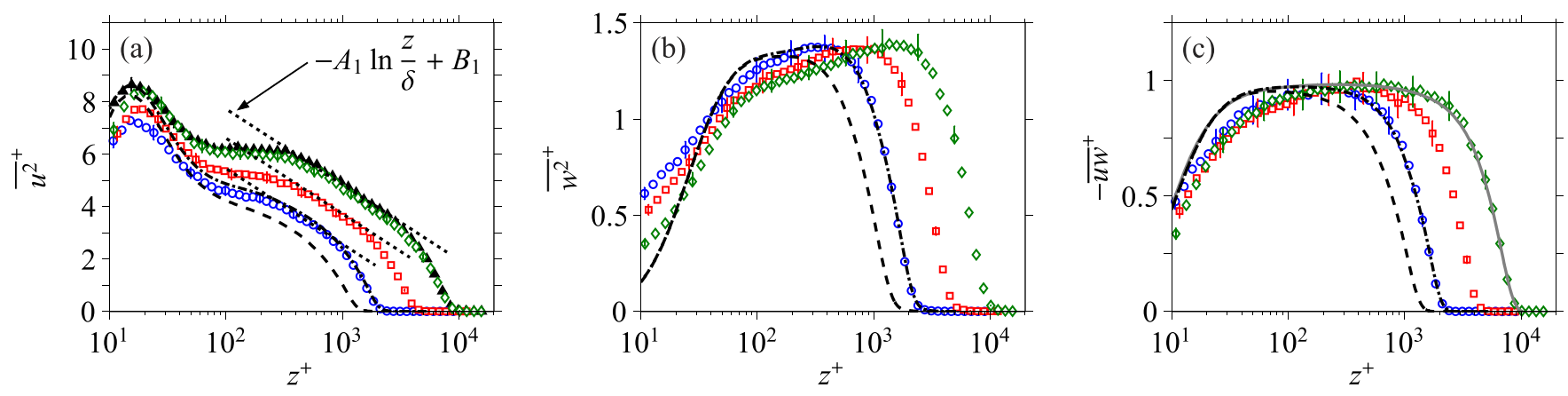

FIG. 2. Profiles of (a) streamwise variance, (b) wall-normal variance, and (c) Reynolds shear stress at multiple Re. Open and solid symbols correspond to X-probe and single hot-wire measurements, where blue circle: $R e_{\tau} \approx 2500$, red square: $R e_{\tau} \approx 5000$, and green diamond, black triangle: $R e_{\tau} \approx 10000$. The vertical lines denote the observed spread in the statistics during the experiment repeats (4\%, 5\%, and $7 \%$ for $\overline{u^{2}}, \overline{w^{2}}$, and $\overline{u w}$, respectively). The dashed and dotted-dashed lines correspond to DNS statistics ${ }^{16}$ at $\delta_{99}^{+}=1310$ and 1990, respectively, while the solid line in (c) denotes the Reynolds shear stress formulation obtained using a composite mean velocity profile ${ }^{14}$ at $R e_{\tau} \approx 10000$.

\section{A. Turbulent stress results}

The streamwise variance $\left(\overline{u^{2}}\right)$, wall-normal variance $\left(\overline{w^{2}}\right)$, and Reynolds shear stress $(-\overline{u w})$ obtained using the $\times$-probe are presented in Figures 2(a)-2(c), respectively. Also shown are profiles obtained by Sillero, Jiménez, and Moser ${ }^{16}$ at $\delta_{99}^{+}$ $=1310$ and 1900 from DNS, as dashed and dotted-dashed lines, respectively (the equivalent Reynolds number is $R e_{\tau} \approx 1700$ and 2500 if $\delta$ based on the composite profile is used instead). At the highest $R e$, a $\overline{u^{2}}$ distribution (denoted by black triangle in Figure 2(a)) obtained using a single-normal hot-wire with $l^{+} \approx 15$ (where $l$ corresponds to the wire length) is also shown, along with a Reynolds shear stress formulation (solid grey line in Figure 2(c)) from the composite fit of Chauhan, Monkewitz, and Nagib ${ }^{14}$ fitted to the measured $U$ profile.

Comparison of the current $R e_{\tau} \approx 2500$ profiles against DNS at a matched $R e$ shows good agreement, at least for $z^{+}>150$. In the region $z^{+}<150, \overline{u^{2}}$ from the $\times$-probe is attenuated due to spatial resolution effects. ${ }^{17,18}$ A good collapse is observed at $R e_{\tau} \approx 10000$ where the single hot-wire and $\times$-probe have comparable spatial resolutions. Furthermore, as evident in Figure 2(a), the $\overline{u^{2}}$ distribution in the inertial sublayer displays a logarithmic relation as a function of $z$. This behaviour is consistent with the $u$ fluctuations obeying the $z$-scaling, as initially predicted by Townsend ${ }^{5}$ using the attached eddy hypothesis. The logarithmic relation ${\overline{u^{2}}}^{+}=-A_{1} \ln (z / \delta)+B_{1}$ (indicated by the dotted line in Figure 2(a)), where $A_{1}=1.25$ as reported by Marusic et al. ${ }^{19}$ and Meneveau and Marusic, ${ }^{20}$ agrees well with the current dataset.

At a fixed $z^{+}$location, a marginal decrease in ${\overline{w^{2}}}^{+}$is observed in Figure 2(b) with increasing $R e$ in the region $z^{+}<500$; however the differences remain less than $5 \%$ of the measured values and are within the experimental uncertainty. Furthermore, the near-wall $R e$ trends from the experiments are not replicated by the profiles from DNS where a nominal collapse of the ${\overline{w^{2}}}^{+}$statistics is observed up to $z^{+}=100$ instead (cf. dashed and dotted-dashed lines in Figure 2(b)). Recent near-wall high resolution Particle Image Velocimetry measurements (with a window size of $5 \times 5$ viscous length scales) performed at $R e_{\tau} \approx 8000$ by de Silva et al. ${ }^{21}$ indicates that the collapse of ${\overline{w^{2}}}^{+}$in this region extends to high $R e$. This suggests that the current dataset suffers from a slight variation in attenuation or amplification of the $\overline{w^{2}}$ statistics across $R e$ (although the spatial resolution is kept nominally constant). These errors are presumed to be related to calibration errors. It should be noted that the transition from amplified to attenuated $\overline{w^{2}}$ recorded by the $\times$-probe compared to DNS at $z^{+} \approx 30$ is characteristic of that observed for this particular $\times$-probe dimension, ${ }^{12}$ which is due to finite sensor separation effects.

A good estimate of the $-\overline{u w}$ profile can be constructed using the mean momentum equation if the $U$ distribution as a function of $z$ and a friction factor relation as a function of the development distance are known. ${ }^{22}$ Hence, the formulation at $R e_{\tau} \approx 10000$ shown in Figure 2(c) has been obtained in such a manner. The challenges associated with measuring an accurate Reynolds shear stress are evident when the direct measurements of $-\overline{u w}$ in the literature ${ }^{23}$ are surveyed, with discrepancy of up to $15 \%$ between the measured and inferred (from the $U$ profile) values common.

\section{SCALING OF THE SPECTRA AND COSPECTRA OF TURBULENT MOTIONS}

Figures 3 and 4 show the $u, w$ spectra, and $u w$ cospectra from multiple $z$ positions at $R e_{\tau} \approx 10000$, pre-multiplied by the streamwise wavenumber, $k_{x}$. Here, $\lambda_{x}=2 \pi / k_{x}$ corresponds to the wavelength of the energetic modes. Furthermore, in the pre-multiplied representation, the total area under the curve when integrated against $\ln k_{x}$ (or $\ln \lambda_{x}$ ) is equal to the turbulent stress. It should be noted that Taylor's frozen turbulence hypothesis ${ }^{24}$ is utilised here to convert the hot-wire signals from the temporal to spatial domain. That is, turbulent motions at all length scales are assumed to convect at a constant velocity that is equal to the local mean velocity. While this is a common practice for hot-wire measurements, strictly speaking the convention velocity is scale dependent, ${ }^{25}$ meaning some scale-redistribution of energy occurs in the present spectra. Furthermore, del Álamo and Jiménez ${ }^{25}$ observe that while the convection velocity is highly scale 

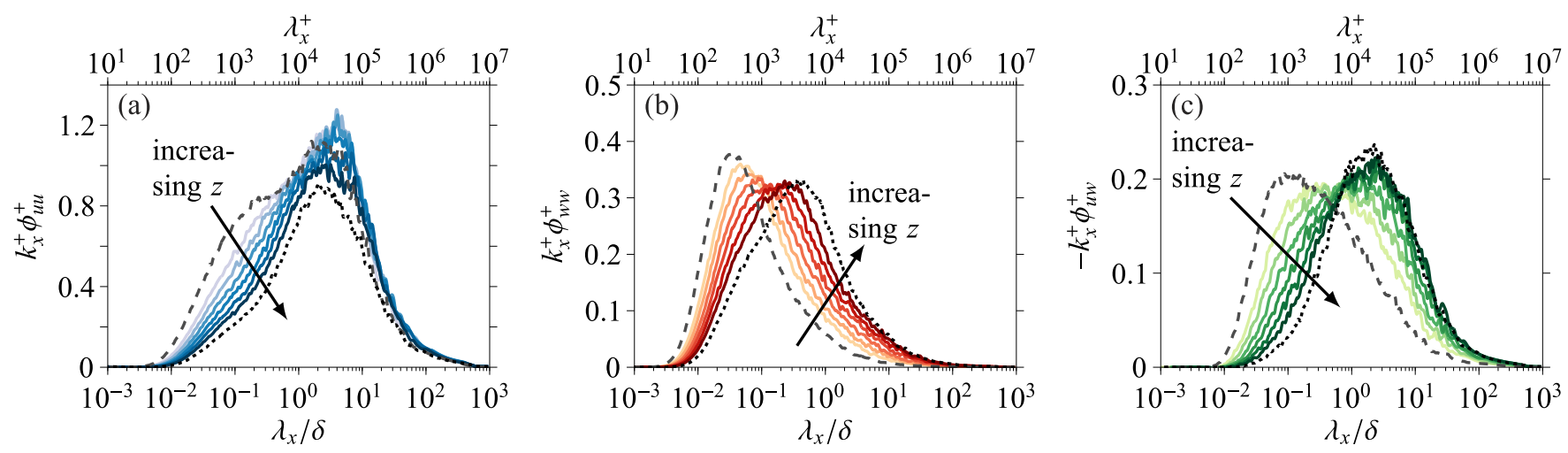

FIG. 3. Pre-multiplied spectra and cospectra at $R e_{\tau} \approx 10000$ for (a) streamwise velocity, (b) wall-normal velocity, and (c) Reynolds shear stress. The scaling of $u w$ cospectra with $z$ is observed at selective wall heights, indicated using solid lines; while dashed $\left(z^{+} \approx 150\right)$ and dotted $\left(z^{+} \approx 0.2 \delta^{+}\right)$lines correspond to locations outside the self-similar region. The arrow indicates the direction of increasing wall position, with the colour shading in (a)-(c) corresponding to the same $z$ location.
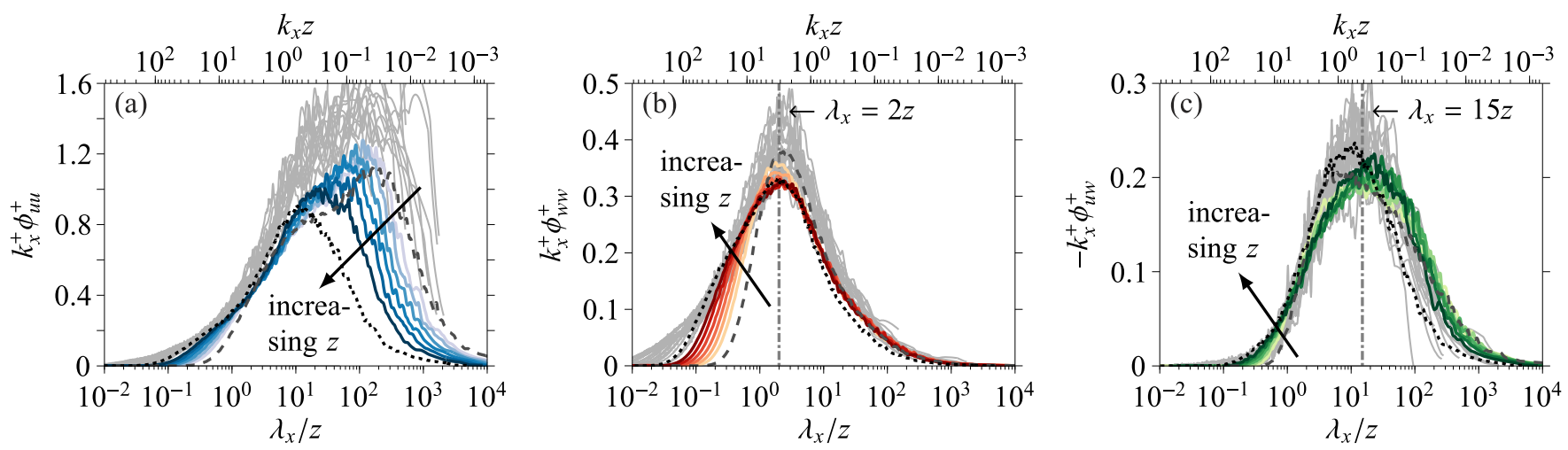

FIG. 4. As Figure 3, however the abscissa is now normalised by the wall-height, $z$. The solid grey lines correspond to spectra from Kunkel and Marusic. ${ }^{27}$

dependent and substantially different from the mean velocity close to the wall, away from the wall (in the inertial region) the local mean velocity provides a good approximation at most energetic scales. Hence, the redistribution of energy in Figure 3 corresponds to a small fraction of the overall energy (accounting for $\sim 5 \%$ uncertainty in the measured spectra ${ }^{25}$ ); however, we cannot entirely rule out its influence here. The abscissa in Figures 3(a)-3(c) is shown normalised in viscous and $\delta$ length scales (here the ratio between the two is a constant since only one $R e$ case is shown). Moreover, the same spectra, but now with $\lambda_{x}$ normalised by the wall height, $z$, are shown in Figure 4.

In $\delta$-scaling (cf. Figure 3), a collapse is only observed for the $u$ velocity and is limited to very large scales $\left(\lambda_{x}>5 \delta\right)$. Meanwhile, Figure 4(a) shows that the $u$ motions exhibit $z$-scaling in the range $\mathcal{O}(1) \lesssim \lambda_{x} / z \lesssim \mathcal{O}(10)$. However, the overlap region where the $z$ and $\delta$-scaling (cf. Figure 3(a)) are simultaneously satisfied is difficult to observe and
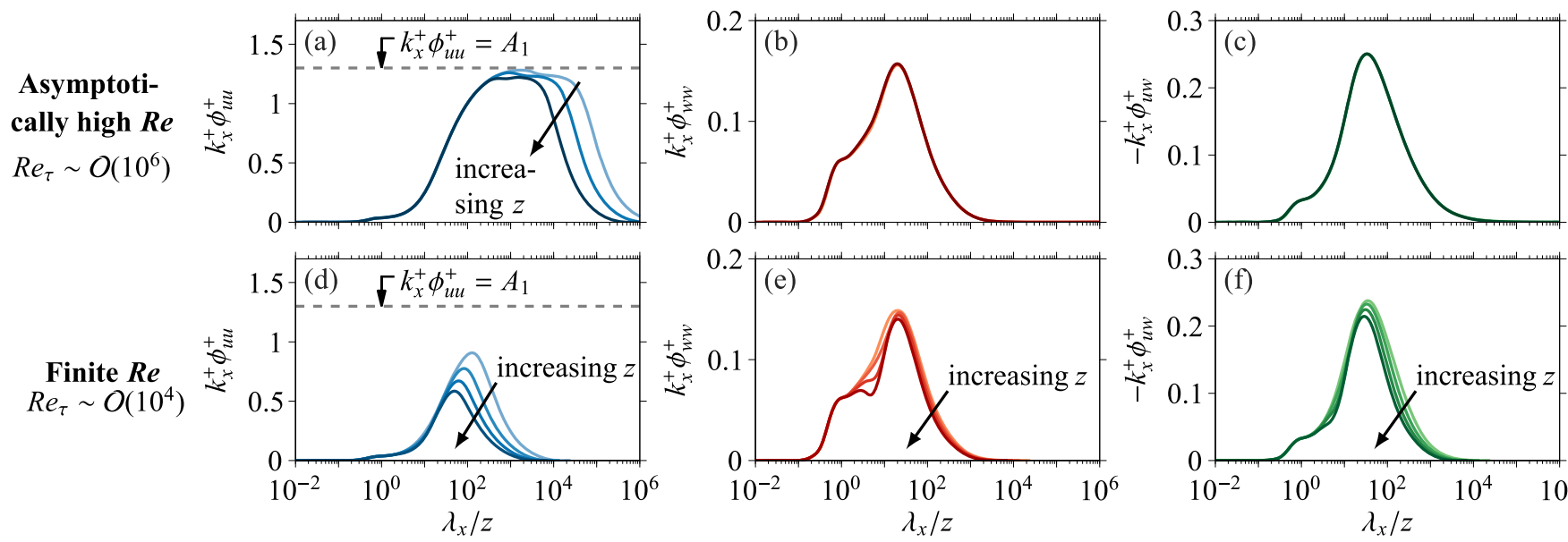

FIG. 5. The expected distributions of the pre-multiplied spectra and cospectra at (a) $-(\mathrm{c})$ an asymptotic state $\left(\operatorname{Re}_{\tau} \sim \mathcal{O}\left(10^{6}\right)\right)$ and $(\mathrm{d})-(\mathrm{f})$ a limited scale separation $\left(R e_{\tau} \sim \mathcal{O}\left(10^{4}\right)\right)$, following the attached eddy hypothesis. Left column ((a) and (d)): streamwise velocity spectra, centre column ((b) and (e)): wall-normal velocity spectra, right column ((b) and (d)): Reynolds shear stress cospectra; shown at varying $z$ locations within the inertial sublayer. 
consequently, no clear plateau region for $k_{x} \phi_{u u}$, corresponding to a $k_{x}^{-1}$ scaling law, is visible. On the other hand, a good collapse of the $w$ and $u w$ motions in $\lambda_{x} \gtrsim \mathcal{O}(z)$ regimes is observed at selective $z$ locations when $\lambda_{x}$ is normalised by $z$ (cf. Figures 4(b) and 4(c)), an indication that the $w$ and $u w$ containing motions in these regions follow a pure $z$-scaling. The dominant modes for the $w$ spectra and $u w$ cospectra occur at $\lambda_{x} \approx 2 z$ and $15 z$, respectively, as indicated in Figures 4(b) and 4(c), and are in good agreement with DNS datasets of Jiménez and Hoyas, ${ }^{26}$ albeit at a lower $R e$.

It should be noted that the analysis presented here is based on data in the region $z^{+} \geq 150$, where the $\overline{w^{2}}$ and $\overline{u w}$ statistics are found to be more robust to the calibration errors. Also shown in Figure 4 are the spectra measured by Kunkel and Marusic ${ }^{27}$ at very high $\operatorname{Re}\left(\operatorname{Re}_{\tau} \sim \mathcal{O}\left(10^{6}\right)\right)$ in the inertial sublayer $\left(z / \delta \sim \mathcal{O}\left(10^{-3}\right)\right)$ from the atmospheric surface layer measurements (shown as solid grey lines). Although a higher degree of uncertainty is introduced due to the challenges associated with measuring in the atmospheric surface layer (which includes estimating $U_{\tau}$ and thus shifting where the solid grey lines are located on the ordinate of Figure 4), similar to our laboratory measurements a good degree of collapse is observed for $\phi_{w w}$ and $\phi_{u w}$ but not for $\phi_{u u}$ across two decades of $R e$ when the wavelength is normalised by the $z$ location.

\section{AN EXPLANATION BASED ON THE PHENOMENOLOGY OF THE ATTACHED EDDY MODEL}

In this section, we attempt to identify the physical mechanism that leads to the $w$ and $u w$ containing motions exhibiting the pure $z$-scaling more readily at a lower $R e$ than the mixed scaling for the $u$ velocity. For this purpose, the attached eddy hypothesis of Townsend ${ }^{5}$ and Perry and Chong ${ }^{6}$ is used to extract the spectra from an idealised flow field where selfsimilarity is strictly enforced. In this frame work, a representative eddy that captures the bulk features of the average contribution from coherent structures with an identical characteristic height, $\mathcal{H}$, is used to construct the flow field. Hence, the flow is modelled as a collection of representative eddies, where each eddy is scaled accordingly by different scaling factors and is then randomly distributed in the plane of the wall. As an illustration, Figures 6(a)-6(d) show a representative eddy packet, together with its contribution to the streamwise velocity, wall-normal velocity, and Reynolds shear stress obtained from Biot-Savart calculations of the vortex rods. ${ }^{28}$ It should be noted that here our aim is not to capture the instantaneous flow features present in the real flow, which are unlikely to resemble the representative eddy, but rather to obtain the bulk statistics contributed by these flow features.

The attached eddy hypothesis is an inviscid model, and hence a finite slip velocity is permitted at the wall. The slip velocity is restricted to the wall-parallel plane, while the wallnormal velocity $(w)$ at the wall is enforced to be zero, to preserve the no-penetration condition. This means that in the vicinity of the wall, the $w$ contribution from the head of the eddy, where the flow is highly vortical (in the spanwise direction), diminishes much more rapidly compared to the other two components; and thus the motions become increasingly restricted to the wall-parallel plane in the near-wall region. Consequently, as shown by Perry and Marusic ${ }^{29}$ (and also evident from Figures 6(b)-6(d)), the $u$ velocity contribution from an attached eddy extends all the way to the wall while the $w$ and $u w$ contributions are restricted to $z \sim \mathcal{H}$. Since the total velocity is a summation of contributions from a range of attached eddy sizes, the largest energetic scale in $u$ increases relative to $z$ as the wall is approached, while the largest $w$ and $u w$ containing motions remain fixed.

\section{A. Attached eddy calculations}

Figures 5(a)-5(f) show results from attached eddy calculations using simple $\Lambda$ eddies in a packet configuration (where multiple eddies align coherently in the streamwise direction) for the representative eddy (cf. Figure 6(a)). The calculations involve integrating the spectral contributions from the representative eddy for each $z$ location, ${ }^{9}$ where the bounds of integration depend on $z$. The top row, Figs. 5(a)-5(c), results correspond to an asymptotic prediction at a very high $\operatorname{Re}\left(\operatorname{Re}_{\tau} \sim \mathcal{O}\left(10^{6}\right)\right)$, while the bottom row, Figs. 5(d)-5(f), corresponds to predictions at $R e$ close to the experiments. The wall heights shown in Figures 5(a)-5(c) correspond to $\mathcal{O}\left(10^{-4}\right) \lesssim z / \delta \lesssim \mathcal{O}\left(10^{-3}\right)$ or $\mathcal{O}\left(10^{2}\right) \lesssim z^{+} \lesssim \mathcal{O}\left(10^{3}\right)$. Hence, the highest $z$ location shown here coincides with that of the atmospheric surface layer measurements shown in Figure 4, while the lowest $z$ location is an order of magnitude closer to the wall and no experimental data from Kunkel and Marusic ${ }^{27}$ exist at this height. It should be noted that the acquisition of the very long time-series is not possible in atmospheric measurements due to the limited periods of neutral stability. Therefore, the spectra for large wavelengths $\left(\lambda_{x} / z \sim \mathcal{O}\left(10^{3}\right)\right)$ have a limited number of ensembles, which leads to an increased uncertainty in the energetic content measured at these wavelengths, and it is not possible to resolve the very long wavelengths. The predictions shown in Figures 5(d)-5(f) are for $\mathcal{O}\left(\sqrt{\delta^{+}}\right) \lesssim z^{+} \lesssim \mathcal{O}\left(0.1 \delta^{+}\right)$, similar to the experimental results which are shown in Figure 4. Although results from only a single representative eddy are shown here, we find that the $R e$ trends exhibited are retained regardless of the representative eddy shape chosen (including a single $\Lambda$ eddy instead of a packet of $\Lambda$ eddies). See the work of Baidya et al. ${ }^{28}$ for details.

\section{B. Spectral behaviour in the asymptotical limit}

Perry, Henbest, and Chong ${ }^{9}$ show that in the asymptotic limit the $u$ spectra are expected to follow $z$ - and $\delta$-scaling. Hence, for a given wall height $z$, all the eddies in the inertial sublayer with height $\gg \mathcal{O}(z)$ contribute an identical energy content to the $u$ velocity, leading to an inversely proportional distribution for $\phi_{u u}$ as a function of the streamwise wavenumbers (i.e., $\phi_{u u} \sim k_{x}^{-1}$ ). The attached eddy calculations indeed exhibit a plateau like behaviour for the pre-multiplied $u$ spectra (the $k_{x}^{-1}$ law appears as a plateau in the pre-multiplied representation) at $R e_{\tau} \sim \mathcal{O}\left(10^{6}\right)$, as shown in Figure 5(a). Furthermore in the asymptotic limit, the plateau approaches a value $A_{1}$ and variation in $\overline{u^{2}}$ as a function of $z$ occurs solely due to an extension of the $k_{x}^{-1}$ plateau region as the wall is approached. ${ }^{9}$ Therefore, $A_{1}$ also corresponds to the slope of 

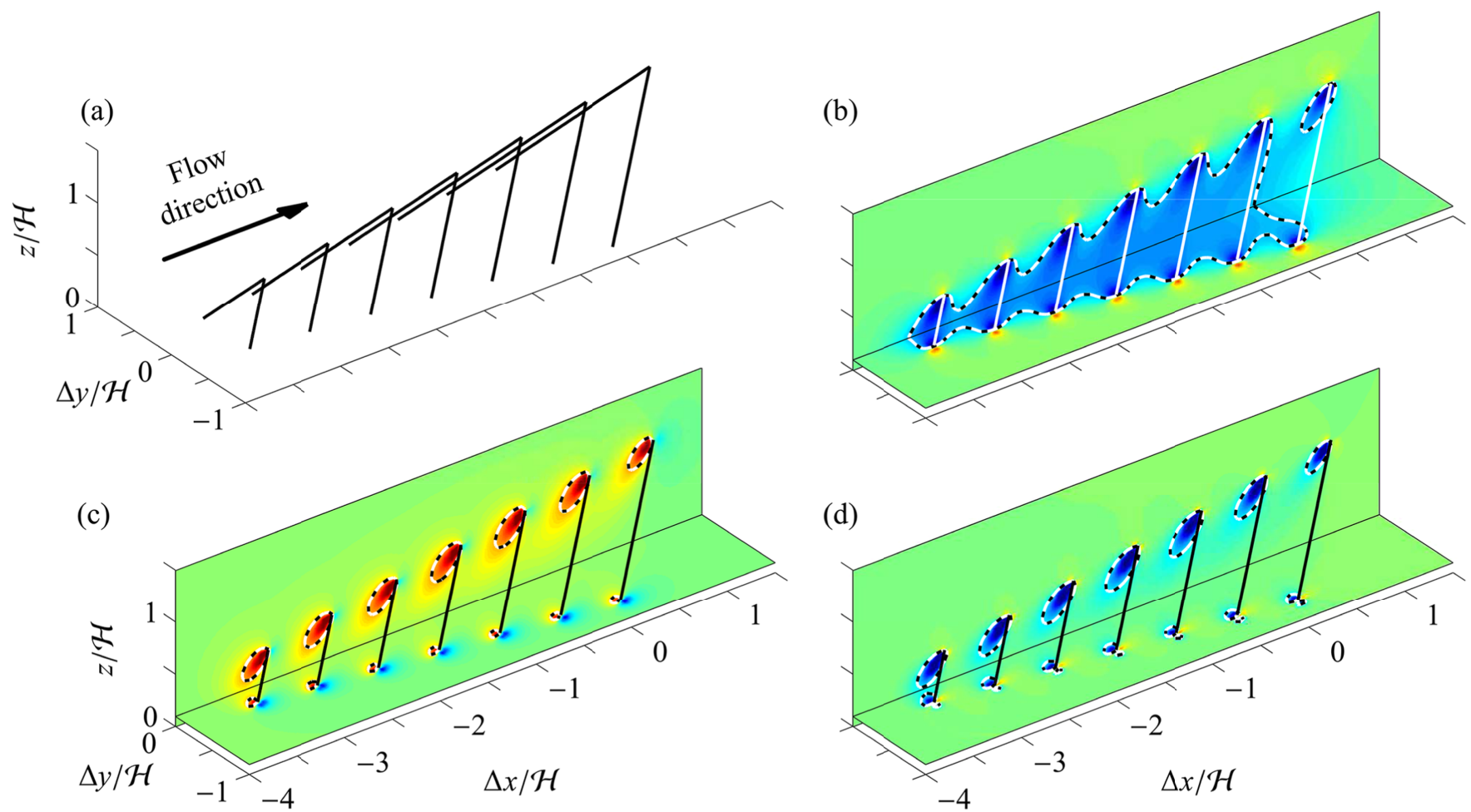

negative

zero

\section{$\Delta u, \Delta w, \Delta u \Delta w$}

FIG. 6. Velocity contributions from a representative eddy. (a) A packet of $\Lambda$ hairpin vortices. Corresponding contributions to (b) streamwise velocity, (c) wallnormal velocity, and (d) Reynolds shear stress. The solid lines denote vortex rods, while dashed lines indicate contours of $\left|\left(\Delta u^{+}\right)^{2}\right|,\left|\left(\Delta w^{+}\right)^{2}\right|,\left|\Delta u^{+} \Delta w^{+}\right|=0.6$ (only the negative $\Delta u$, the positive $\Delta w$, and the negative $\Delta u \Delta w$ contours are shown).

the logarithmic behaviour in $\overline{u^{2}}$ with respect to $z$ (cf. Figure 2(a)). On the other hand, the $w$ spectra and the $u w$ cospectra at multiple $z$ positions remain identical in terms of $\lambda_{x} / z$, as shown in Figures 5(b) and 5(c), since unlike the $u$ spectra they only exhibit $z$-scaling. It should be noted that the attached eddy model used here does not account for fine-scale detached eddies that exist in the real flow. ${ }^{29}$ These additional contribution from the detached eddies is responsible for deviations from $z$-scaling observed in the $u$ and $w$ spectra for the $\lambda_{x}<z$ regime, evident in Figures 4(a) and 4(b). Furthermore, the finescale detached eddies are near isotropic ${ }^{29,30}$ with a minimal contribution to the Reynolds shear stress. Thus, a good collapse of the $u w$ cospectra in $z$-scaling still holds for the $\lambda_{x}<z$ regime (cf. Figure 4(c)). Here, only a brief description of the attached eddy hypothesis relevant to the present work is provided, and the reader is referred to Ref. 9 and Ref. 29 for a full description.

\section{Finite $R e$ effects}

One of the requirements for the $k_{x}^{-1}$ law in the $u$ spectra is the existence of a range of scales which simultaneously satisfy $z$ - and $\delta$-scaling, where the energetic content across the $z$ positions collapses when $\lambda_{x}$ is normalised by $z$ and $\delta$, respectively. ${ }^{9}$ Hence, even when the entire $u$ contributions are from eddies that scale with $z$, such as the case for the attached eddy calculations shown in Figure 5(d), a plateau region in $k_{x} \phi_{u u}$ will not necessarily eventuate. This is due to the inadequate scale separation existing between the smallest and largest length scales at a finite $R e$, and hence no wall location exists where $z \ll \delta$ such that the $u$ contributions from the eddies of height $\sim \mathcal{O}(\delta)$ are universal irrespective of $z$, while also simultaneously being sufficiently far away from the wall to be fully inertial $\left(z \gg U_{\tau} / v\right)$. Furthermore, in a real boundary layer velocity contributions from eddies that do not follow the attached eddy hypothesis do exist (e.g., fine-scale detached eddies $^{9}$ and superstructures ${ }^{31,32}$ ), and these additional contributions can lead to further departure from the ideal $k_{x}^{-1}$ law behaviour in the $u$ spectra.

Conversely, the wall-normal velocity and Reynolds shear stress contributions from the energetic eddies are localised to $z$ positions close to the characteristic eddy height. This is because the blocking effect of the wall means that the motions become increasingly constrained in the wall-parallel plane, with minimal $w$ (and subsequently modest $-u w$ ) contribution, as the wall is approached. Hence, the $w$ contributions from the eddies that do not scale with the wall height make up a small portion of the cumulative $\overline{w^{2}}$ and $-\overline{u w}$, unlike the $u$ contributions to the $\overline{u^{2}}$ statistic. Therefore, the wall-scaling is more evident in the $w$ and $u w$ containing motions or the active region 
of the flow. This leads to $\phi_{w w}$ and $\phi_{u w}$ that resemble the selfsimilar asymptotic state even at a finite $R e$, as demonstrated in Figures 5(e) and 5(f). Therefore, the empirical observation that $\phi_{w w}$ and $\phi_{u w}$ resemble the asymptotic state, even when the scale separation present in the flow is insufficient to exhibit the $k_{x}^{-1}$ law in the $u$ spectra, is consistent with the attached eddy hypothesis.

Figures 5(e) and 5(f) show that at a finite $R e$, a small (compared to the $u$ spectra) but nevertheless persistent deviation occurs in the $w$ spectra and $u w$ cospectra with increasing $z$ locations, and that these deviations are larger in the $u w$ cospectra than in the $w$ spectra. These differences arise from the no penetration condition that exists at the wall, and hence in the vicinity of the wall, the attached eddy contributions follow $\overline{w^{2}} \propto(z / \mathcal{H})^{2}$ and $-\overline{u w} \propto z / \mathcal{H}$ relationships. ${ }^{5}$ Here $\mathcal{H}$ corresponds to the characteristic height of the eddy. A good agreement between Figures 4(b), 4(c), 5(e), and 5(f) suggests that the experimental $w$ spectra and $u w$ cospectra can be well described in terms of the $z$-scaling and finite $R e$ effects. Further, the $u w$ cospectra are harder to converge than the $w$ spectra due to the highly intermittent nature of instantaneous Reynolds shear stress contributions. Hence, we can expect a larger scatter in the measured $u w$ cospectra compared to the $w$ spectra (for a given sample size and duration), which leads to larger deviations existing between multiple $u w$ cospectra measurements compared to the $w$ spectra. Inspection of the $w$ spectra and $u w$ cospectra from experiments, shown in Figures 4(b) and 4(c) (where both have been computed using an identical routine), indeed indicates that the $w$ spectra are smoother and therefore better converged than the $u w$ cospectra.

\section{STREAMWISE, WALL-NORMAL SPECTROGRAMS, AND REYNOLDS SHEAR STRESS COSPECTROGRAM}

Figure 7 shows pre-multiplied $\phi_{u u}, \phi_{w w}$, and $\phi_{u w}$, plotted against the wall distance and wavelength for various $R e$. For the $w$ spectrogram (cf. Figures 7(d)-7(f)), the inertial sublayer corresponds to a region where the contours follow the $\lambda_{x} \propto z$ lines, with the most dominant energy density occurring at $\lambda_{x} \approx 2 z$ (shown as a dotted-dashed line in Figures 7(d)-7(f)). Therefore, unlike the $u$ spectrogram (cf. Figures 7(a)-7(c)) which exhibits two distinct dominant energetic sites, ${ }^{31,32}$ the $w$ spectrogram is ridge-like with near constant $w$ contribution across all $z$ locations residing in the inertial sublayer.

For the $u w$ cospectrogram, two distinct peaks are observed outside the self-similar region at $z^{+} \approx 50, \lambda_{x}^{+} \approx 1000$ and
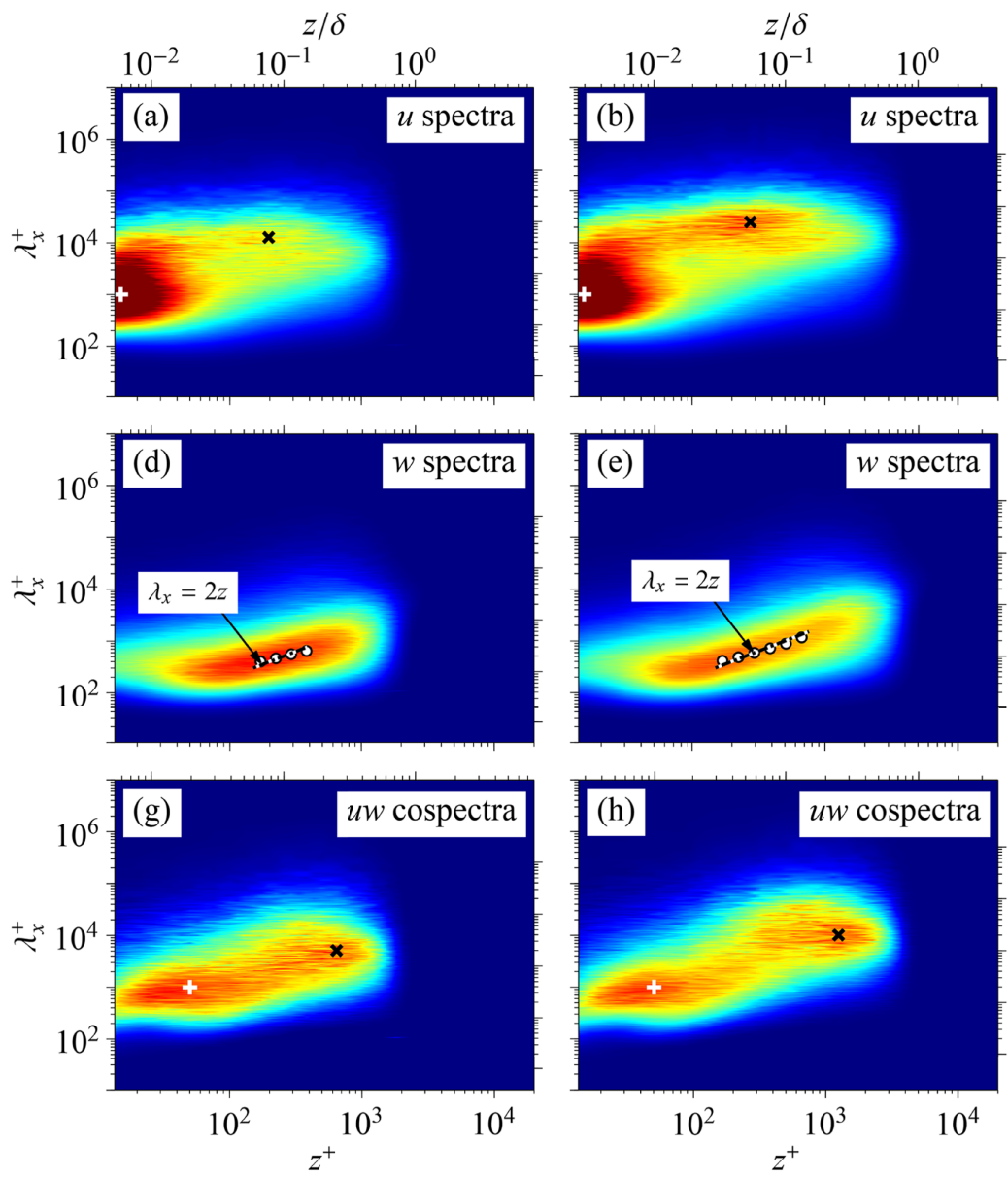
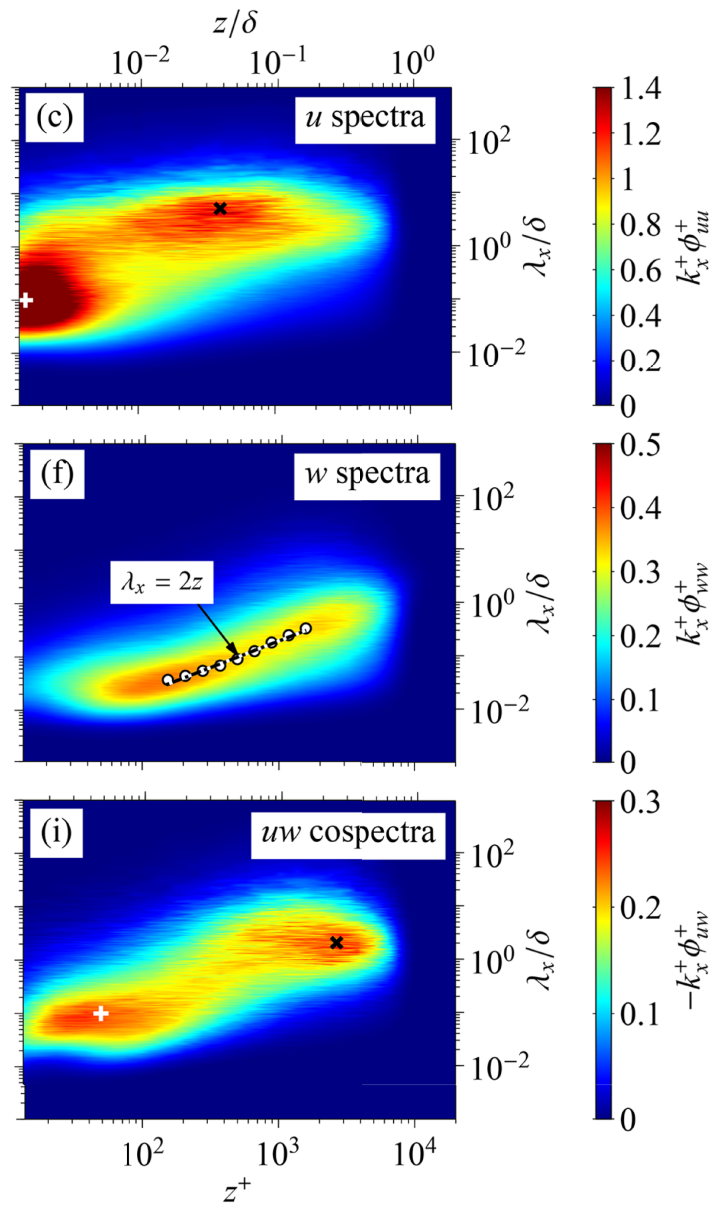

FIG. 7. Pre-multiplied spectrograms and cospectrogram at (a), (d), and (g): $R e_{\tau} \approx 2500$, (b), (e), and (h): $R e_{\tau} \approx 5000$, and (e), (f), and (i): $R e_{\tau} \approx 10000$. Top row $((\mathrm{a})-(\mathrm{c}))$ : streamwise velocity spectra, middle row ((d)-(f)): wall-normal velocity spectra, and bottom row ((g)-(i)): Reynolds shear stress cospectra. In (d)-(f), the circles indicate the location of the peak $k_{x} \phi_{w w}$ contributions at a particular wall-height, while the dotted-dashed lines correspond to the relationship $\lambda_{x}=2 z$; both within the region $150 \leq z^{+} \leq 0.15 \delta^{+}$. Symbols + and $\times$denote the location of two dominant energetic sites in the $u$ spectrogram and $u w$ cospectrogram. 
$z / \delta \approx 0.25, \lambda_{x} / \delta \approx 2$, and is denoted using symbols + and $\times$, respectively, in Figures 7(g)-7(i). These peaks are thought to be associated with the inner and outer peak in the $u$ spectrogram (cf. Figures 7(a)-7(c)) and therefore arise due to the near-wall cycle and very large-scale motions ${ }^{33}$ or superstructures. $^{31,32}$ Thus, the peak associated with the large-scale contributions moves outwards with $R e$ in viscous units as shown in Figures 7(g)-7(i). Furthermore, we find that similar to the $u$ spectra, both the $w$ spectra and the $u w$ cospectra display $R e$ invariance for the small-scale motions in the near-wall region $\left(\lambda_{x}^{+} \lesssim \mathcal{O}\left(10^{4}\right)\right.$ and $\left.z^{+} \lesssim \mathcal{O}\left(10^{2}\right)\right)$ when scaled in viscous units, and thus it is highly likely that a single physical mechanism (i.e., the near-wall cycle) is responsible for these energetic $u$ and $w$ contributions.

\section{A. Limits of the inertial sublayer region}

Classically, the inertial sublayer is thought to start at a fixed viscous unit away from the wall (typically $z^{+} \sim 100$ ) and end at a fraction of the boundary layer thickness ${ }^{3,34}$ $(z / \delta \sim 0.15)$. Indeed, the available experimental data suggest that the mean streamwise velocity profile follows a logarithmic law in the inertial sublayer. ${ }^{19}$ However, as higher Re data have become available, the start of the inertial sublayer reported has also increased, with $z^{+}=600$ documented at a very high $R e$ regime in the Superpipe facility. ${ }^{35}$

More recently, Wei et al. ${ }^{1}$ have argued that rather than considering balance of stresses, as is the case in the classical view, the gradient of stresses is the significant quantities since they appear in the mean momentum equation (see also Ref. 2 for a concise review). Subsequently, Wei et al. ${ }^{1}$ showed that contributions due to the viscous stress gradient are significant starting from the wall to the $z$ location where the peak Reynolds shear stress occurs. They also inferred that the start of the inertial sublayer scales as $z^{+} \sim \sqrt{\delta^{+}}$. Furthermore, the available literature ${ }^{36,37}$ suggests that the $z$ location where the maximum $-\overline{u w}$ occurs moves outwards under viscous scaling with increasing $R e$. Following these developments, Marusic et al. ${ }^{19}$ simultaneously re-examined the universality of the log law in both the $U$ and $\overline{u^{2}}$ statistics with respect to $z$, as predicted by $z$-scaling using very high $R e$ experiments. This allowed them to overcome the difficultly in determining the $z$ limits of inertial sublayer solely using a $U$ profile, which deviates very slowly from the $\log$ law. Consequently, they find that $z^{+} \sim \sqrt{\delta^{+}}$ scaling is consistent with the starting $z$ location where the log behaviour in $U$ and $\overline{u^{2}}$ is simultaneously fulfilled.

Here, in order to determine the $z$ limits of the inertial sublayer, we will utilise the attached eddy results from Sec. IV, which suggest that a good collapse of the entire energetic $\overline{w^{2}}$ and $\overline{u w}$ contents occur in the inertial sublayer when scaled with $z$. In other words, the deviations observed from the asymptotic state serve as a measure to determine the $z$ locations where self-similarity holds. Although for a synthetic flow with a perfect $z$-scaling, the $w$ spectra were shown to exhibit the best collapse and hence provide the most accurate assessment of self-similarity, in the real flow an additional contribution from fine-scale detached eddies that are not accounted for in the perfect $z$-scaling model exists (cf. Figures 4(b) and 5(b)) as illustrated in Figure 8. However, these fine-scale detached eddies are near isotropic, ${ }^{29}$ and hence their contribution to the Reynolds shear stress is minimal. Thus, for the $u w$ cospectra, the entire energy-containing range exhibits the $z$-scaling in the inertial sublayer, making it the most appropriate quantity (out of all the turbulent stresses) to provide an assessment of self-similarity.

\section{Assessment of the deviations from self-similarity}

In order to quantify deviations from the self-similarity, we will therefore utilise the quantity $\Delta k_{x} \phi_{u w}$, which is defined as

$$
\Delta k_{x} \phi_{u w}\left(z, \lambda_{x}\right)=k_{x} \phi_{u w}\left(z, \lambda_{x}\right)-k_{x} \phi_{u w}\left(z_{\text {ref }}, \lambda_{x} z_{\text {ref }} / z\right),
$$

where $z_{\text {ref }}$ is located at the geometric centre of the selfsimilar region. In other words, $\Delta k_{x} \phi_{u w}$ corresponds to a difference between the measured $u w$ cospectra (cf. Figure 9(a)) and a $u w$ cospectrogram where the wall self-similarity (i.e., $z$-scaling) is satisfied across the entire boundary layer (cf. Figure $9(\mathrm{~b})$ and the term $k_{x} \phi_{u w}\left(z_{\text {ref }}, \lambda_{x} z_{\text {ref }} / z\right)$ in (1)), as illustrated in Figure 9. This procedure iteratively determines the appropriate limits for the start $\left(z_{i}\right)$ and end $\left(z_{o}\right)$ of the

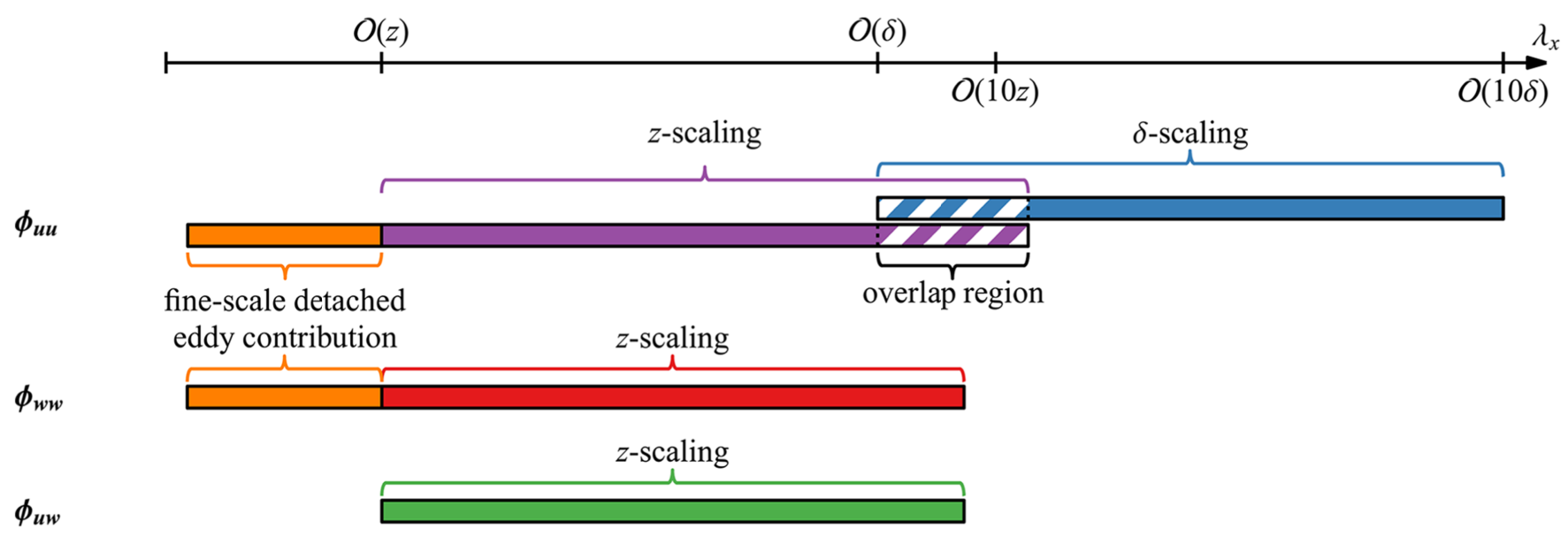

FIG. 8. A summary of various scaling for turbulent stress spectra and cospectra in the inertial sublayer at high $R e$. The overlap region in the $u$ spectra leads to a $\bar{u}^{2} \sim-A_{1} \ln (z / \delta)+B_{1}$ behaviour. 

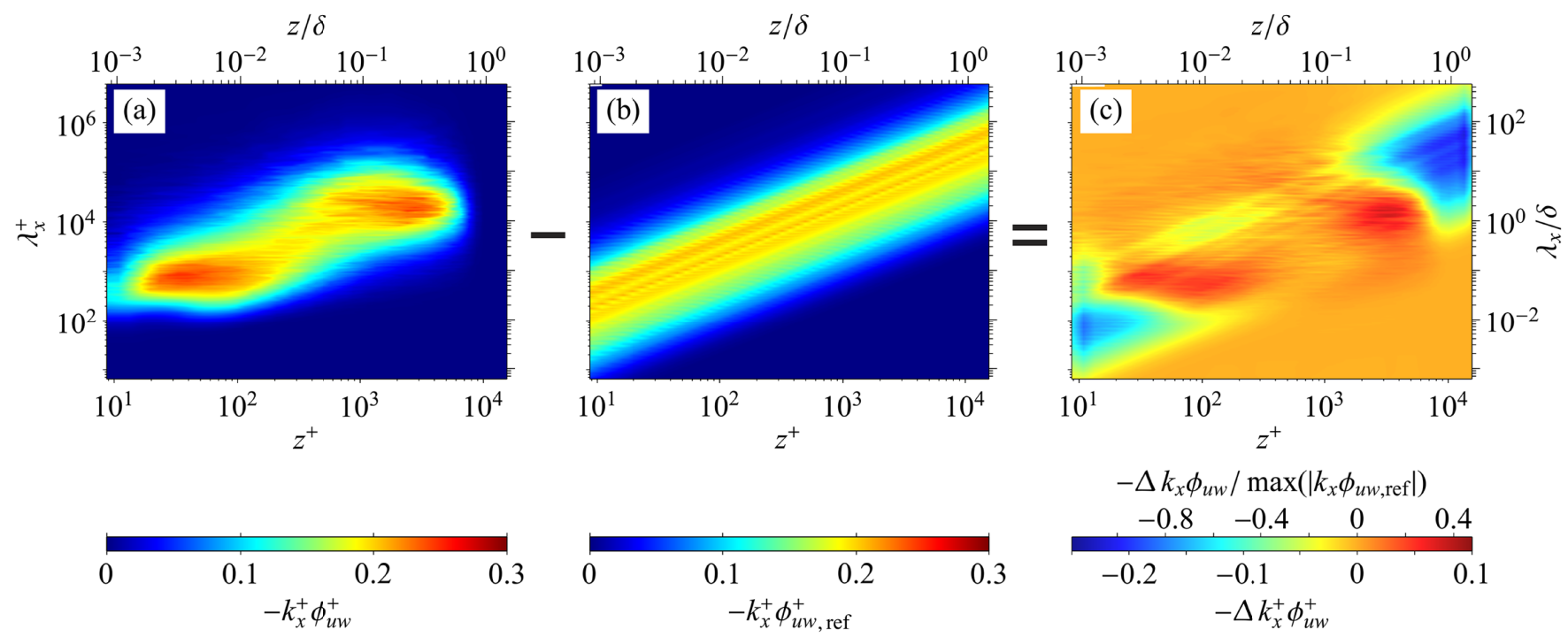

FIG. 9. Assessment of the self-similarity using deviation from a perfect $z$-scaling for $R e_{\tau} \approx 10000$. (a) $u w$ cospectra from the experiments, (b) a reference $u w$ cospectrum satisfying a perfect $z$-scaling, and (c) differences between (a) and (b).

inertial sublayer (and hence $z_{\text {ref }}$, since $z_{\text {ref }}=\sqrt{z_{i} z_{o}}$ ) such that the $u w$ cospectra within the limits $z_{i}<z<z_{0}$ satisfy the $\Delta k_{x} \phi_{u w}\left(z, \lambda_{x}\right) \simeq 0$ condition. Furthermore, regardless of the initial $z_{i}$ and $z_{o}$ guesses, the procedure is observed to converge to the same $z$ limits.

Although the quantity $\Delta k_{x} \phi_{u w}$ shown in Figure 9(c) is sufficient for an assessment of self-similarity, some of $\Delta k_{x} \phi_{u w} \simeq 0$ regions in the figure are due to near zero $u w$ content at a particular $z$ and $\lambda_{x}$ location (e.g., $z^{+}=10^{4}$ and $\lambda_{x}^{+}=10^{2}$ ). Hence, in Figures 10(a)-10(c), the contour maps of $-\Delta k_{x} \phi_{u w}$ have been weighted by the $-k_{x} \phi_{u w}$ value to enhance the differences, $\Delta k_{x} \phi_{u w}$, where the $u w$ contribution is dominant. In other words, $u w$ cospectra as functions of $z$ and $\lambda_{x}$ (cf. Figures $7(\mathrm{~g})-7(\mathrm{i})$ ) are used to blend the conventional one dimensional colour map (such as the one used in Figure 9(c)) from the original to white depending on how the $-k_{x} \phi_{u w}$ value changes from the maximum value to zero to create a two dimensional colour map. For example, at $\left(z^{+}, \lambda_{x}^{+}\right) \approx\left(50,10^{3}\right)$ and $\left(10^{4}, 10^{2}\right)$, the pre-multiplied Reynolds shear stress cospectra are near the maximum and zero, respectively, and therefore while the first location retains the non-modified colour, the second location is near white.
The markers 1-3 and A-B in Figures 10(a)-10(c) denote regions of low and high Reynolds shear stress compared to the self-similar asymptotic state, respectively. Also shown are contours corresponding to $-\Delta k_{x}^{+} \phi_{u w}^{+}=-0.035$ and 0.035 as dotted-dashed and dotted lines, used here as a criteria to determine the $z$-limits of the inertial sublayer. Here, the threshold selected corresponds to the estimated uncertainty in the measured spectrogram, which is equal to the maximum deviation from a polynomial curve with respect to $\log \lambda_{x}$ used to fit the measured spectra. Furthermore, although the coefficients (i.e., 3 and 0.15 , corresponding to the vertical dashed lines in Figure 10) are dependent on the threshold used, the $z^{+} \sim \sqrt{\delta^{+}}$and $\delta^{+}$ scaling for the start and end of the self-similar region are, respectively, retained.

The regions 1 and 2 correspond to locations where $-\overline{u w}$ is no longer constant, leading to a negative $-\Delta k_{x} \phi_{u w}$. These regions remain fixed in viscous and outer units, respectively, as shown in Figures 10(a)-10(c). Meanwhile, the regions of positive $-\Delta k_{x} \phi_{u w}$, A and $\mathrm{B}$, are associated with the peak in the $u w$ cospectra and hence they also scale with viscous and outer scaling, correspondingly. Finally, at an increased $R e$, an additional region with insufficient Reynolds shear stress
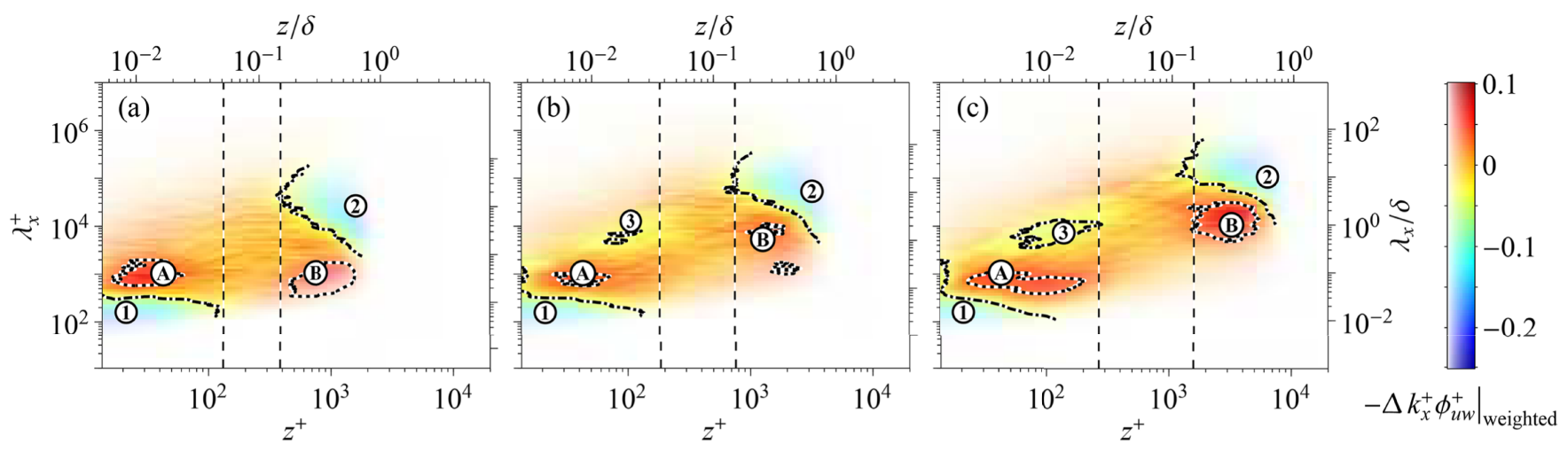

FIG. 10. Deviations in the weighted Reynolds shear stress cospectra $\left(-\left.\Delta k_{x}^{+} \phi_{u w}^{+}\right|_{\text {weighted }}\right.$ ) from the geometric centre of the inertial sublayer at (a) $R e_{\tau} \approx 2500$, (b) $R e_{\tau} \approx 5000$, and (c) $R e_{\tau} \approx 10000$. The dotted-dashed and dotted lines denote contour levels at -0.035 and 0.035 , while the vertical dashed lines indicate locations $z^{+} \sim 3 \sqrt{\delta^{+}}$and $0.15 \delta^{+}$. 
contribution (denoted by 3 ) compared to the self-similarity state emerges below the inertial sublayer, leading to $z^{+} \sim \sqrt{\delta^{+}}$ scaling for the start of the inertial sublayer. The relatively large streamwise length scales associated with the region 3 compared to $z$ and the fact that the corresponding $w$ spectra for the region exhibit a near perfect $z$-scaling (cf. $\lambda_{x}>10 z$ regime in Figure 4(b)) suggest that this region arises from the direct influence of the large $\delta$-scaled features in the $u$ spectra which become increasingly prominent at high $R e$. Interestingly, region 3 is also related to the $\delta$-scaled vortex-stretching motions. ${ }^{38}$ Furthermore it should be noted that the attached eddy hypothesis ${ }^{6}$ predicts a $-\overline{u w}^{+}=1-\left(\frac{z}{\delta}\right) \varepsilon$ distribution, with the constant $\varepsilon \ll 1$, for the Reynolds shear stress in the inertial sublayer. This means that the $u w$ cospectra increasingly deviate from the perfect $z$-scaling with increasing $z$, even in the inertial sublayer, and this may be the reason why the region 2 start to encroach the $z / \delta=0.15$ line with increasing $R e$ as observed in Figure 10(c). However, with an increase in $R e$, a higher portion of $z$ locations satisfies $-\overline{u w}^{+} \simeq 1$, since the start of the inertial region approaches zero in the $z / \delta$ normalisation. We also find that, in the inertial sublayer, the integral $-\int_{0}^{\infty} \Delta k_{x}^{+} \phi_{u w}^{+} \mathrm{d} \ln k_{x}^{+}$decreases with increasing $z$, which is consistent with the mean momentum balance ${ }^{1}$ and the attached eddy hypothesis. ${ }^{6}$

From Figures 10(a)-10(c), it is evident that although the $u w$ cospectra depart from self-similarity observed in the regions $z^{+}<3 \sqrt{\delta^{+}}$; the dominant energetic mode in the $w$ spectra still exhibit the $\lambda_{x} \propto z$ relation much closer to the wall. In fact, the wall location where the dominant $w$ mode starts obeying $z$-scaling is fixed in viscous units $\left(z^{+} \approx 100\right)$ for all three $R e$ cases examined here as shown in Figures 7 (d)-7(f). Thus, although self-similarity is only retained for $3 \sqrt{\delta^{+}} \lesssim z^{+} \lesssim 0.15 \delta^{+}$, the observation suggests that the eddies which are responsible for the turbulent stresses begin to exhibit scaling with the distance from the wall at a fixed $z$ location in viscous units, as initially postulated by Perry, Henbest, and Chong. ${ }^{9}$ However, below $z^{+}<3 \sqrt{\delta^{+}}$, the eddies become distorted and depart from self-similarity since the viscous force remains a leading order contribution in the mean momentum equation in this region.

\section{SUMMARY AND CONCLUSIONS}

We demonstrate that in the inertial sublayer, the $u$ velocity scales both with $z$ and $\delta$, therefore exhibiting a mixed scaling. Meanwhile, a major fraction (> 80\%) of $\overline{w^{2}}$ containing motions scale with respect to $z$, with the rest of contribution occurring from near isotropic fine-scale detached eddies. Furthermore, since the near isotropic fine-scales and very large $\delta$-scaled motions have negligible contribution to the Reynolds shear stress, a pure $z$-scaling is observed in the $u w$ cospectra across the range of energetic scales. These scaling laws are also illustrated in Figure 8.

We find that the $w$ spectra and the $u w$ cospectra both follow the self-similar asymptotic state more closely compared to the $u$ spectra at the same scale separation. This is because the scale separation between $z$ and $\delta$ affects the behaviour of the $u$ spectra much more severely than for $\phi_{w w}$ and $\phi_{u w}$. Hence, the flow consisting of self-similar eddies do not necessarily need to exhibit the asymptotical $k_{x}^{-1}$ prediction in the $u$ spectra at a finite $R e$, and this is demonstrated in this paper through the use of the attached eddy framework. ${ }^{5,6}$

The experimental data presented adhere to the trends predicted using the attached eddy hypothesis at limited scale separation, namely, no plateau region in pre-multiplied $u$ spectra but collapse of $w$ spectra and $u w$ cospectra when the wavelength is normalised by the wall height at selective $z$ locations. We also show that the structures with dominant $w$ contributions exhibit $z$-scaling, starting from $z^{+} \sim \mathcal{O}(100)$ and ending at $\mathcal{O}\left(0.1 \delta^{+}\right)$. However, the self-similar region (a near perfect $z$-scaling of $u w$ cospectra) is constrained to $3 \sqrt{\delta^{+}} \lesssim z^{+} \lesssim 0.15 \delta^{+}$; and thus the start of the inertial sublayer is found to change in viscous units, in contrast to the classical view. The $z^{+} \sim \sqrt{\delta^{+}}$scaling for the start of the inertial sublayer is due to the viscous forces becoming a leading order contribution in the mean momentum balance below this region, which leads to the distortion of eddies with height $<3 \sqrt{\delta^{+}}$(in viscous units), and hence the departure from self-similarity.

\section{ACKNOWLEDGMENTS}

This paper is dedicated in the memory of John Lumley, whose work and legacy has had a profound impact on shaping the thoughts and research directions of the authors. The personal interactions with I.M. will always be treasured. The authors also wish to acknowledge the financial support of this research by the Australian Research Council.

${ }^{1}$ T. Wei, P. Fife, J. Klewicki, and P. McMurtry, "Properties of the mean momentum balance in turbulent boundary layer, pipe and channel flows," J. Fluid Mech. 522, 303-327 (2005).

${ }^{2}$ J. C. Klewicki, "Reynolds number dependence, scaling, and dynamics of turbulent boundary layers," J. Fluids Eng. 132, 094001 (2010).

${ }^{3}$ H. Tennekes and J. L. Lumley, A First Course in Turbulence (MIT Press, 1972).

${ }^{4}$ L. Prandtl, "The mechanics of viscous fluids," in Aerodynamic Theory, edited by W. F. Durand (Springer, Berlin, 1935), Vol. III, pp. 34-208.

${ }^{5}$ A. A. Townsend, The Structure of Turbulent Shear Flow (Cambridge University Press, Cambridge, UK, 1976).

${ }^{6}$ A. E. Perry and M. S. Chong, "On the mechanism of wall turbulence," J. Fluid Mech. 119, 173-217 (1982).

${ }^{7}$ J. C. del Álamo, J. Jiménez, P. Zandonade, and R. D. Moser, "Self-similar vortex clusters in the turbulent logarithmic region," J. Fluid Mech. 561, 329-358 (2006).

${ }^{8}$ J. C. Klewicki, "Self-similar mean dynamics in turbulent wall flows," J. Fluid Mech. 718, 596-621 (2013).

${ }^{9}$ A. E. Perry, S. Henbest, and M. S. Chong, "A theoretical and experimental study of wall turbulence,” J. Fluid Mech. 165, 163-199 (1986).

${ }^{10}$ P. A. Davidson, T. B. Nickels, and P. A. Krogstad, “The logarithmic structure function law in wall-layer turbulence," J. Fluid Mech. 550, 51-60 (2006).

${ }^{11}$ R. Baidya, J. Philip, N. Hutchins, J. P. Monty, and I. Marusic, "Measurements of streamwise and spanwise fluctuating velocity components in a high Reynolds number turbulent boundary layer," in Proceedings of 18th Australasian Fluid Mechanics Conference, 2012.

${ }^{12}$ R. Baidya, "Multi-component velocity measurements in turbulent boundary layers," Ph.D. thesis, University of Melbourne, 2016.

${ }^{13}$ T. B. Nickels, I. Marusic, S. Hafez, and M. S. Chong, "Evidence of the $k_{1}^{-1}$ law in a high-Reynolds-number turbulent boundary layer," Phys. Rev. Lett. 95, 074501 (2005).

${ }^{14}$ K. A. Chauhan, P. A. Monkewitz, and H. M. Nagib, "Criteria for assessing experiments in zero pressure gradient boundary layers," Fluid Dyn. Res. 41, 021404 (2009).

${ }^{15}$ N. Hutchins, T. B. Nickels, I. Marusic, and M. S. Chong, "Hot-wire spatial resolution issues in wall-bounded turbulence," J. Fluid Mech. 635, 103-136 (2009). 
${ }^{16}$ J. A. Sillero, J. Jiménez, and R. D. Moser, "One-point statistics for turbulent wall-bounded flows at Reynolds numbers up to $\delta^{+} \approx 2000$," Phys. Fluids 25, 105102 (2013).

${ }^{17}$ Y. Suzuki and N. Kasagi, "Evaluation of hot-wire measurements in wall shear turbulence using a direct numerical simulation database," Exp. Therm. Fluid Sci. 5, 69-77 (1992).

${ }^{18} \mathrm{P}$. Burattini and R. A. Antonia, "The effect of different $\times$-wire calibration schemes on some turbulence statistics," Exp. Fluids 38, 80-89 (2005).

${ }^{19}$ I. Marusic, J. P. Monty, M. Hultmark, and A. J. Smits, "On the logarithmic region in wall turbulence," J. Fluid Mech. 716, 716 (2013).

${ }^{20} \mathrm{C}$. Meneveau and I. Marusic, "Generalized logarithmic law for highorder moments in turbulent boundary layers," J. Fluid Mech. 719, R1 (2013).

${ }^{21}$ C. M. de Silva, E. P. Gnanamanickam, C. Atkinson, N. A. Buchmann, N. Hutchins, J. Soria, and I. Marusic, "High spatial range velocity measurements in a high Reynolds number turbulent boundary layer," Phys. Fluids 26, 025117 (2014).

${ }^{22}$ J. C. Rotta, "Turbulent boundary layers in incompressible flow," Prog. Aerosp. Sci. 2, 1-219 (1962).

${ }^{23}$ H. H. Fernholz and P. J. Finley, "The incompressible zero-pressure-gradient turbulent boundary layer: An assessment of the data," Prog. Aerosp. Sci. 32, 245-311 (1996).

${ }^{24}$ G. I. Taylor, “The spectrum of turbulence,” Proc. R. Soc. A 164, 476-490 (1938).

${ }^{25}$ J. C. del Álamo and J. Jiménez, "Estimation of turbulent convection velocities and corrections to Taylor's approximation," J. Fluid Mech. 640, 5-26 (2009).

${ }^{26}$ J. Jiménez and S. Hoyas, "Turbulent fluctuations above the buffer layer of wall-bounded flows,” J. Fluid Mech. 611, 215-236 (2008).
${ }^{27}$ G. J. Kunkel and I. Marusic, "Study of the near-wall-turbulent region of the high-Reynolds-number boundary layer using an atmospheric flow," J. Fluid Mech. 548, 375-402 (2006).

${ }^{28}$ R. Baidya, J. Philip, J. P. Monty, N. Hutchins, and I. Marusic, "Comparisons of turbulence stresses from experiments against the attached eddy hypothesis in boundary layers," in Proceedings of 19th Australasan Fluid Mechanics Conference, 2014.

${ }^{29}$ A. E. Perry and I. Marusic, "A wall-wake model for the turbulence structure of boundary layers. Part 1. Extension of the attached eddy hypothesis," J. Fluid Mech. 298, 361-388 (1995).

${ }^{30}$ S. G. Saddoughi and S. V. Veeravalli, "Local isotropy in turbulent boundary layers at high Reynolds number," J. Fluid Mech. 268, 333-372 (1994).

${ }^{31}$ N. Hutchins and I. Marusic, "Large-scale influences in near-wall turbulence," Philos. Trans. R. Soc., A 365, 647-664 (2007).

${ }^{32}$ N. Hutchins and I. Marusic, "Evidence of very long meandering features in the logarithmic region of turbulent boundary layers," J. Fluid Mech. 579, 1-28 (2007).

${ }^{33}$ K. C. Kim and R. J. Adrian, "Very large-scale motion in the outer layer," Phys. Fluids 11, 417-422 (1999).

${ }^{34}$ S. B. Pope, Turbulent Flows (Cambridge University Press, 2000).

${ }^{35}$ M. V. Zagarola and A. J. Smits, "Mean-flow scaling of turbulent pipe flow," J. Fluid Mech. 373, 33-79 (1998).

${ }^{36}$ K. R. Sreenivasan, "The turbulent boundary layer," in Frontiers in Experimental Fluid Mechanics (Springer, 1989), pp. 159-209.

${ }^{37}$ M. H. Buschmann, T. Indinger, and M. Gad-el-Hak, "Near-wall behavior of turbulent wall-bounded flows," Int. J. Heat Fluid Flow 30, 993-1006 (2009).

${ }^{38}$ C. Chin, J. Philip, J. Klewicki, A. Ooi, and I. Marusic, "Reynolds-numberdependent turbulent inertia and onset of $\log$ region in pipe flows," J. Fluid Mech. 757, 747-769 (2014). 